\title{
Catastrophic alpha quenching alleviated by helicity flux and shear
}

\author{
A. Brandenburg ${ }^{1}$ and C. Sandin ${ }^{2}$ \\ 1 NORDITA, Blegdamsvej 17, 2100 Copenhagen $\varnothing$, Denmark \\ e-mail: brandenb@nordita.dk \\ 2 Astrophysical Institute Potsdam, An der Sternwarte 16, 14482 Potsdam, Germany \\ e-mail: CSandin@aip.de
}

Received 16 January 2004 / Accepted 21 July 2004

\begin{abstract}
A new simulation set-up is proposed for studying mean field dynamo action. The model combines the computational advantages of local Cartesian geometry with the ability to include a shear profile that resembles the sun's differential rotation at low latitudes. It is shown that in a two-dimensional mean field model this geometry produces cyclic solutions with dynamo waves traveling away from the equator - as expected for a positive alpha effect in the northern hemisphere. In three dimensions with turbulence driven by a helical forcing function, an alpha effect is self-consistently generated in the presence of a finite imposed toroidal magnetic field. The results suggest that, due to a finite flux of current helicity out of the domain, alpha quenching appears to be non-catastrophic - at least for intermediate values of the magnetic Reynolds number. For larger values of the magnetic Reynolds number, however, there is evidence for a reversal of the trend and that $\alpha$ may decrease with increasing magnetic Reynolds number. Control experiments with closed boundaries confirm that in the absence of a current helicity flux, but with shear as before, alpha quenching is always catastrophic and alpha decreases inversely proportional to the magnetic Reynolds number. For solar parameters, our results suggest a current helicity flux of about $0.001 \mathrm{G}^{2} / \mathrm{s}$. This corresponds to a magnetic helicity flux, integrated over the northern hemisphere and over the 11 year solar cycle, of about $10^{46} \mathrm{Mx}^{2}$.
\end{abstract}

Key words. magnetohydrodynamics (MHD) - turbulence - Sun: magnetic fields

\section{Introduction}

The large scale magnetic field of stars and galaxies is often interpreted in terms of mean field dynamo theory, which takes into account that the turbulence is at least partially helical. The helicity, in turn, leads to the so-called $\alpha$ effect, i.e. an averaged field-aligned current that induces new field loops perpendicular to the original field (Moffatt 1978; Krause \& Rädler 1980). In the presence of shear, poloidal loops get sheared out, thereby reinforcing the toroidal field from which more poloidal loops can be created. This is the basic $\alpha \Omega$ dynamo mechanism which is at least in principle able to explain the cyclic variation of the solar magnetic field (Parker 1979).

The investigation of mean field dynamos has been the subject of numerous papers since the 1970s (see also Zeldovich et al. 1983). With the advent of high resolution turbulence simulations it has become exceedingly clear that there is a serious problem in the nonlinear case at large magnetic Reynolds numbers. Similar problems are completely unknown in the context of nonmagnetic, purely hydrodynamic turbulence or in nonhelical hydromagnetic turbulence where the associated dissipative fluxes always remain finite. This is not the case with the magnetic helicity flux which goes to zero in the large magnetic Reynolds number limit (Berger 1984). The magnetic helicity is thus almost perfectly conserved in practically all astrophysically interesting cases.

An important consequence of magnetic helicity conservation is the fact that the $\alpha$ effect cannot produce any net magnetic helicity. This means that if the $\alpha$ effect produces large scale magnetic fields, it must at the same time also give rise to a certain amount of small scale fields with opposite sign of magnetic helicity (Seehafer 1996; Ji 1999). Hence the strength of the large scale field that can be generated on dynamical time scales is limited, as the associated small scale field cannot grow significantly above the equipartition field strength (Brandenburg 2001, hereafter B01; Field \& Blackman 2002; Blackman \& Brandenburg 2002; Subramanian 2002). Recent work has shown that this leads to a rather restrictive nonlinearity of the $\alpha$ effect. It has been recognized for some time (Blackman \& Field 2000a,b; Kleeorin et al. 2000) that the conservation of magnetic helicity may be particularly restrictive in the presence of closed or periodic boundary conditions used in many investigations. While it is clear that magnetic helicity fluxes through boundaries can help in principle (Brandenburg et al. 2002), one must still ensure that it also is of the right properties. If the magnetic helicity flux carries away most of the desired large scale field, nothing will be gained and the dynamo will be even less efficient. This is indeed what early simulations have shown (Brandenburg \& Dobler 2001). 


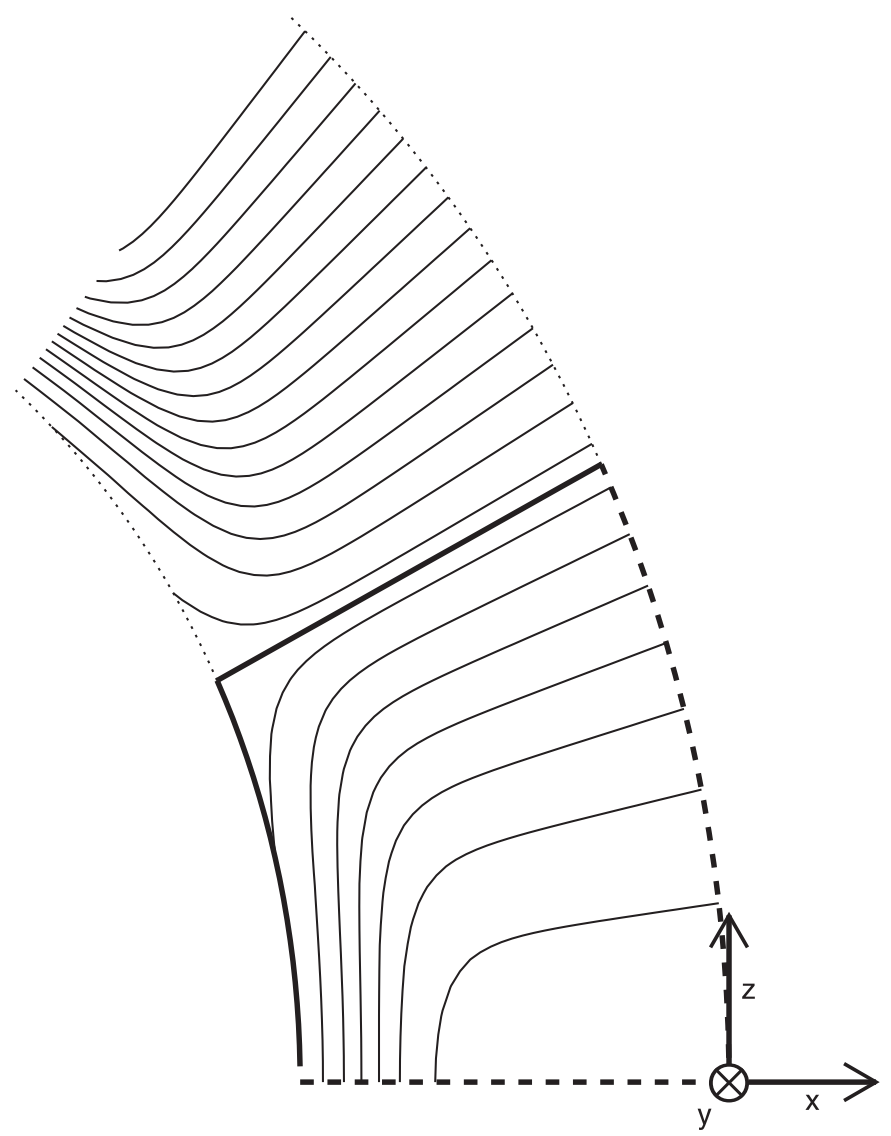

Fig. 1. A sketch of the solar angular velocity at low latitudes with spoke-like contours in the bulk of the convection zone merging gradually into uniform rotation in the radiative interior. The low latitude region, modeled in this paper, is indicated by thick lines.

The purpose of the present paper is to show that the situation changes considerably when helicity flux is mediated by shear, the only mechanism known that can separate and hence also transport magnetic helicity in space (the $\alpha$ effect, by comparison, separates and transports magnetic helicity in wavenumber space).

We adopt the simplest possible model that is able to capture the effects of helicity transport from one hemisphere to the other. To motivate our model we first look at an idealized representation of the solar angular velocity which is spoke-like in the bulk of the convection zone and nearly rigid in the radiative interior; see Fig. 1.

We model the region below $30^{\circ}$ latitude by adopting a Cartesian geometry where the $x$ direction corresponds to radius, the $y$ direction to longitude, and the $z$ direction to latitude. We ignore the fact that in the sun the radial transition to uniform rotation is much steeper and model the mean toroidal velocity simply in terms of trigonometric functions using

$\overline{\boldsymbol{U}}=U_{0} \cos k_{1} x \cos k_{1} z$

where $k_{1}$ is the lowest wavenumber in the $(x, z)$ plane with $-\pi / 2 \leq k_{1} x \leq 0$ and $0 \leq k_{1} z \leq \pi / 2$. In the following we adopt units where $k_{1}=1$. The equator is assumed to be at $z=0$ and the outer surface at $x=0$. The bottom of the convection zone is at $x=-\pi / 2$ and the latitude where the surface angular

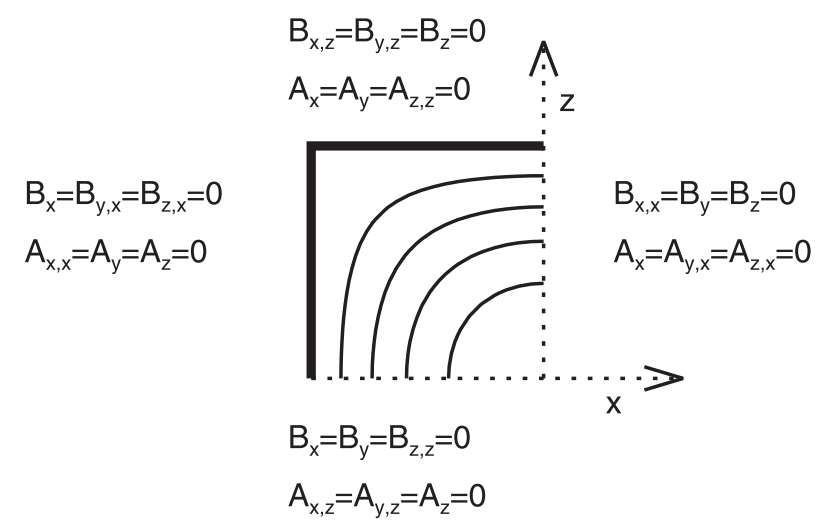

Fig. 2. Differential rotation in our Cartesian model, with the equator being at the bottom, the surface to the right, the bottom of the convection zone to the left and mid-latitudes at the top. The boundary conditions for the three components of the magnetic field and the vector potential (indicated near the boundaries of the box) are discussed in Sect. 2.

velocity equals the value in the radiative interior is at $z=\pi / 2$; see Fig. 2.

In order to clarify some basic properties of this rather unexplored geometry we begin by studying $\alpha \Omega$ dynamos in this geometry in Sect. 3, after introducing our numerical approach in Sect. 2. The problem is at first reduced to two dimensions. Here we assume that there is an $\alpha$ effect so that a mean magnetic field can readily be generated by this term. Next we drive helical turbulence by a corresponding forcing term in three dimensions and measure the resulting $\alpha$ effect by imposing a toroidal magnetic field in Sect. 4. In particular, we study the dependence of $\alpha$ quenching on the magnetic Reynolds number and show that the $\alpha$ effect is only catastrophically quenched when the boundaries are closed or when the large scale shear has been turned off.

\section{Our numerical approach}

The evolution of the magnetic field, $\boldsymbol{B}$, is governed by the induction equation, $\partial \boldsymbol{B} / \partial t=-\boldsymbol{\nabla} \times \boldsymbol{E}$, where $\boldsymbol{E}$ is the electric field. The induction equation is solved in terms of the magnetic vector potential, $\boldsymbol{A}$, where $\boldsymbol{B}=\boldsymbol{\nabla} \times \boldsymbol{A}$. The evolution of $\boldsymbol{A}$ is therefore given by $\partial \boldsymbol{A} / \partial t=-\boldsymbol{E}-\boldsymbol{\nabla} \phi$, where $\phi$ is the scalar potential. By using the gauge in which $\phi=0$ we simply have

$\partial \boldsymbol{A} / \partial t=-\boldsymbol{E}$

which is the equation that will be considered throughout. The (negative) electric field is given by

$-\boldsymbol{E}=\boldsymbol{U} \times \boldsymbol{B}-\eta \mu_{0} \boldsymbol{J}$,

where $\boldsymbol{U}$ is the velocity, $\boldsymbol{J}=\boldsymbol{\nabla} \times \boldsymbol{B} / \mu_{0}$ the current density, and $\mu_{0}$ the vacuum permeability.

At the bottom of the convection zone and at mid-latitudes we assume the same boundary conditions for the magnetic field as for the velocity field, i.e. the field is tangent to the boundaries, which corresponds to perfect conductor boundary conditions. This means that the tangential electric field vanishes, and, because of the gauge $\phi=0$, we have

$A_{x}=A_{y}=0 \quad$ on $z=\pi / 2, \quad$ and 
$A_{y}=A_{z}=0 \quad$ on $x=-\pi / 2$.

On the equator and at the outer surface we assume that the magnetic field is normal to the boundaries, i.e.

$B_{x}=B_{y}=0 \quad$ on $z=0, \quad$ and

$B_{y}=B_{z}=0 \quad$ on $x=0$.

At the equator this boundary condition is consistent with dipolar parity of the field. The full set of boundary conditions for all three components of both $\boldsymbol{A}$ and $\boldsymbol{B}$ are given on the four "meridional" boundaries of the box in Fig. 2. In the $y$ direction we adopt periodic boundary conditions over the interval $0<y<2 \pi$.

Occasionally we refer to the boundary conditions (6) and (7) as open, because they permit flux of magnetic and current helicities through the $z=0$ and $x=0$ boundaries. For comparison we also perform calculations with closed boundaries where $A_{x}=A_{y}=0$ on $z=0$ and $A_{y}=A_{z}=0$ on $x=0$. With these conditions the magnetic and current helicity fluxes vanish through these boundaries. At the bottom of the convection zone and at mid-latitudes the fluxes of magnetic and current helicities are always vanishing. This is probably a reasonable assumption, because at these locations there is no shear to mediate large scale helicity transport and the small scale helicity transport was already previously found to fluctuate around zero if there is no shear (Brandenburg \& Dobler 2001).

For both the mean field calculations and the turbulence simulations we step the equations forward in time by using the Pencil Code ${ }^{1}$. For the mean field calculations a typical resolution of $32^{2}$ meshpoints proved to be sufficient, while for the turbulence simulations the required resolution depends on the magnetic Reynolds number, $R_{\mathrm{m}}$. Here, $R_{\mathrm{m}}$ is based on the magnitude of the turbulent velocity and not on the larger shear flow velocity. (The precise definition is given below in Sect. 4.2.) For $R_{\mathrm{m}} \approx 100$, a resolution of $512^{3}$ meshpoints is required, while for $R_{\mathrm{m}} \approx 15$, a resolution of $128^{3}$ meshpoints proved to be sufficient. We note, however, that the aspect ratio of the box is $1: 4: 1$ and, although there is shear smearing out structures in the $y$ direction, a uniform mesh aspect ratio seems often to be preferred. For example, a run with $128 \times 512 \times 128$ meshpoints allowed us to use higher Reynolds numbers than $128^{3}$ meshpoints.

\section{Mean field calculations}

An important aspect of our studies is to show that the shear flow depicted in Fig. 2 is a reasonable approximation to the differential rotation present in the sun (which is more like that depicted in Fig. 1).

In the context of mean field theory it is known that in spherical shells both dipolar and quadrupolar solutions are approximately equally easily excited (Roberts 1972) and that both solutions can be oscillatory with field migration away from the midplane (when $\alpha>0$ ). We want to know whether in the present geometry the magnetic field evolution is similar to that in spherical shells.

\footnotetext{
${ }^{1}$ http://www.nordita.dk/software/pencil-code
}

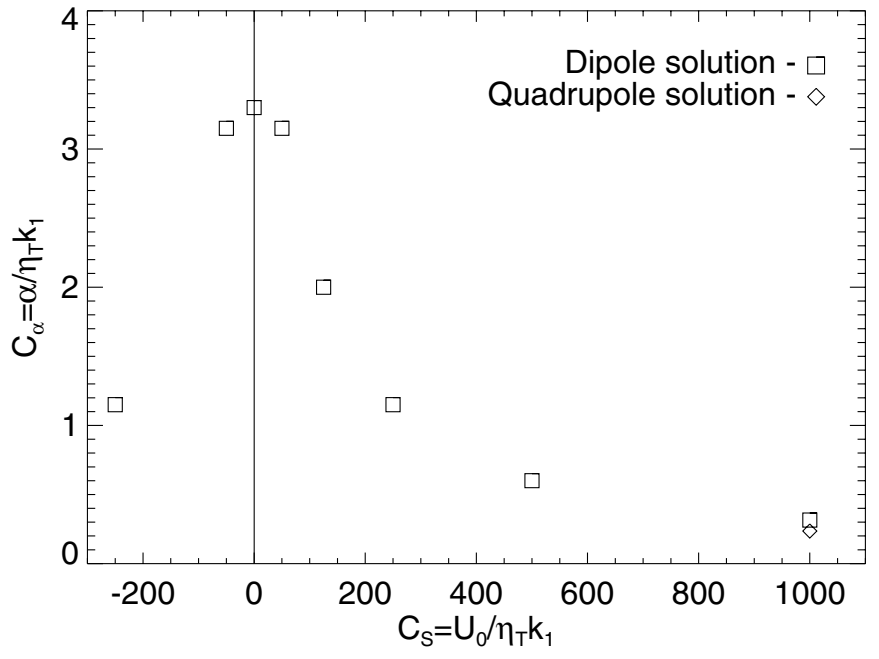

Fig. 3. Critical value of $C_{\alpha}$ for dynamo action as a function of shear parameter, $C_{\mathrm{S}}$. Note the typical decrease of the critical value of $C_{\alpha}$ with increasing $C_{\mathrm{S}}$.

The mean field induction equation is given by $\partial \overline{\boldsymbol{B}} / \partial t=$ $-\boldsymbol{\nabla} \times \overline{\boldsymbol{E}}$, which again is solved in terms of the mean magnetic vector potential, i.e. $\partial \overline{\boldsymbol{A}} / \partial t=-\overline{\boldsymbol{E}}$, where

$-\overline{\boldsymbol{E}}=\overline{\boldsymbol{U}} \times \overline{\boldsymbol{B}}+\overline{\mathcal{E}}-\eta \mu_{0} \overline{\boldsymbol{J}}$.

Here, overbars denote azimuthal averages, and velocity and magnetic field are split into mean and fluctuating components via $\boldsymbol{B}=\overline{\boldsymbol{B}}+\boldsymbol{b}$ and $\boldsymbol{U}=\overline{\boldsymbol{U}}+\boldsymbol{u}$. The electromotive force from the fluctuating components of velocity and magnetic field, $\overline{\boldsymbol{\varepsilon}}=\overline{\boldsymbol{u} \times \boldsymbol{b}}$, is in its simplest form (e.g. Moffatt 1978; Krause \& Rädler 1980)

$\overline{\mathcal{E}}=\alpha \overline{\boldsymbol{B}}-\eta_{\mathrm{t}} \mu_{0} \overline{\boldsymbol{J}}$

where $\alpha$ (related to the mean helicity) and $\eta_{\mathrm{t}}$ (turbulent diffusivity) could still be functions of $x, z$, and $t$, as well as a function of $\overline{\boldsymbol{B}}$ itself, but for simplicity we assume them to be constant here.

The solutions are characterized by two non-dimensional parameters,

$C_{\alpha}=\alpha /\left(\eta_{\mathrm{T}} k_{1}\right), \quad$ and $\quad C_{\mathrm{S}}=U_{0} /\left(\eta_{\mathrm{T}} k_{1}\right)$,

where $\eta_{\mathrm{T}}=\eta+\eta_{\mathrm{t}}$ is the sum of microscopic and turbulent magnetic diffusivities.

In Fig. 3 we plot the stability diagram in the $\left(C_{\alpha}, C_{\mathrm{S}}\right)$ plane. For $C_{\alpha}<C_{\alpha, \text { crit }}$ the solutions are decaying and for $C_{\alpha}>C_{\alpha, \text { crit }}$ they are growing exponentially and are oscillatory (Hopf bifurcation), except for a narrow interval around $C_{\mathrm{S}}=0$. Such a behavior is quite typical of $\alpha \Omega$ dynamos (see, e.g., Roberts \& Stix 1972). For $C_{\mathrm{S}}=1000$ we have also considered the quadrupolar solution and find that it is slightly easier to excite (see Fig. 3). As stated earlier, the approximately equal excitation conditions for dipolar and quadrupolar solutions, seen in Fig. 3, is typical of $\alpha \Omega$ dynamos in spherical shells. Indeed, the fact that quadrupolar solutions can be preferred has been found in other solar dynamo models (Dikpati \& Gilman 2001).

In Fig. 4 we show contours of $\bar{B}_{y}$ for the marginally excited case with $C_{\mathrm{S}}=1000$ for different times covering a little 

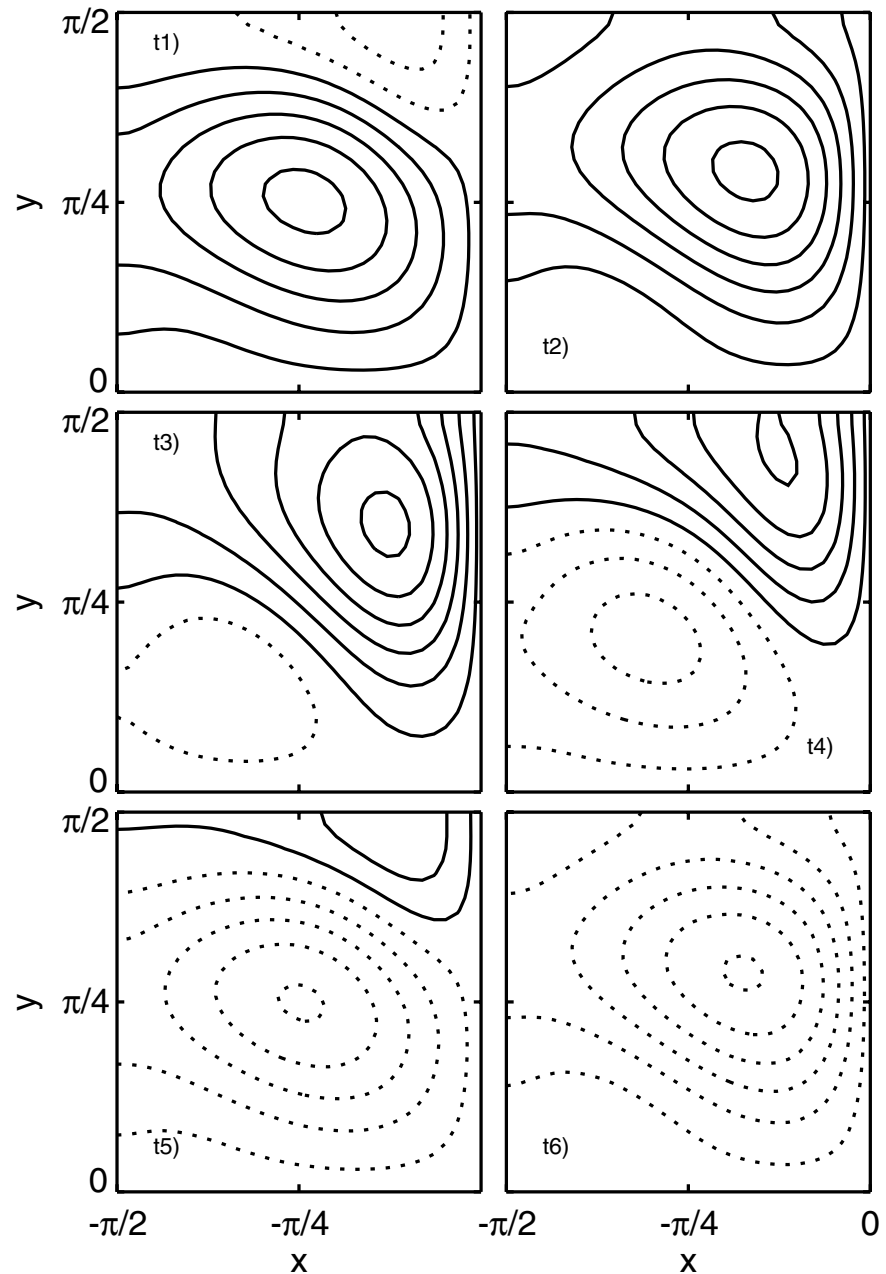

Fig. 4. Contours of $\bar{B}_{y}$ for times separated by $\Delta t=300$ in the temporal sequence t1-t6. Note the field migration from the lower left corner to the upper right; in this figure $C_{\mathrm{S}}=1000$ (see Eq. (10)).

more than half a cycle. One clearly sees magnetic field migration away from the equator. We note that in the sun the field migration is toward the equator. The reason for this is not entirely clear, but it could be caused by a negative $\alpha$ effect (but the reason for this is not clear either) or by meridional circulation (Choudhuri et al. 1995; Durney 1995). However, before these questions can seriously be addressed, it is mandatory to have a reliable mean field theory. This has so far been hampered by not being able to model the nonlinear feedback correctly.

Based on the similarity of the stability diagram and the migration pattern in calculations using Cartesian geometry and spherical shells (e.g. Roberts \& Stix 1972), we may conclude that our local model provides a reasonable approximation to the more realistic case of a spherical shell. Since azimuthal averages are used, the mean field equations would be axisymmetric and could easily be solved using meshpoint methods. The case of three-dimensional turbulence in spherical shells is considerably more difficult because the coordinate singularity at the poles leads to serious timestep restrictions in the azimuthal direction.

\section{Turbulence simulations}

In this section we consider three-dimensional turbulence in the same Cartesian geometry that we used in the previous section. We now consider the full (non-averaged) velocity and magnetic fields, $\boldsymbol{U}$ and $\boldsymbol{B}$, respectively.

\subsection{Basic equations}

The fluctuating velocity together with the shear flow must be obtained by simultaneously solving the induction equation (Eq. (2)) together with the momentum equation, which we write here for an isothermal gas of constant sound speed $c_{\mathrm{s}}$,

$\frac{\mathrm{D} \boldsymbol{U}}{\mathrm{D} t}=-c_{\mathrm{s}}^{2} \boldsymbol{\nabla} \ln \rho+\frac{\boldsymbol{J} \times \boldsymbol{B}}{\rho}+\boldsymbol{f}+\boldsymbol{F}_{\mathrm{visc}}$,

where $f$ is the forcing function driving both the turbulence (around a narrow band of wavenumbers around $k_{\mathrm{f}}=5$ ) and the shear flow (cf. Brandenburg et al. 2001). Moreover,

$\boldsymbol{F}_{\text {visc }}=v\left(\nabla^{2} \boldsymbol{U}+\frac{1}{3} \boldsymbol{\nabla} \boldsymbol{\nabla} \cdot \boldsymbol{U}+2 \mathbf{S} \cdot \boldsymbol{\nabla} \ln \rho\right)$

is the viscous force where $v=$ const is the kinematic viscosity, $\mathrm{S}_{i j}=\frac{1}{2}\left(U_{i, j}+U_{j, i}\right)-\frac{1}{3} \delta_{i j} U_{k, k}$ the traceless rate of strain tensor, and $\rho$ the density which obeys the continuity equation which we solve in the form

$\frac{\mathrm{D} \ln \rho}{\mathrm{D} t}=-\boldsymbol{\nabla} \cdot \boldsymbol{U}$

where $\mathrm{D} / \mathrm{D} t=\partial / \partial t+\boldsymbol{U} \cdot \boldsymbol{\nabla}$ is the advective derivative. We adopt a random forcing function with finite helicity; see B01 for details.

\subsection{Results for $\alpha$ quenching}

We have carried out a range of simulations for different values of the magnetic Reynolds number,

$R_{\mathrm{m}}=u_{\mathrm{rms}} /\left(\eta k_{\mathrm{f}}\right)$,

for both open and closed boundary conditions. (Here, $u_{\mathrm{rms}}$ does not include the mean shear flow.) In order to measure $\alpha$, a uniform magnetic field, $\boldsymbol{B}_{0}=$ const., is imposed, and the magnetic field is now written as $\boldsymbol{B}=\boldsymbol{B}_{0}+\boldsymbol{\nabla} \times \boldsymbol{A}$. In Fig. 5 we show a graphical presentation of a typical snapshot of a solution.

We have determined $\alpha$ by measuring the turbulent electromotive force, i.e. $\alpha=\langle\mathcal{E}\rangle \cdot \boldsymbol{B}_{0} / B_{0}^{2}$. Similar investigations have been done before both for forced turbulence (e.g., Cattaneo \& Hughes 1996, see also B01) and for convective turbulence (e.g., Brandenburg et al. 1990; Ossendrijver et al. 2001). The contribution from $\eta_{\mathrm{T}} \bar{J}$ has been ignored in this approach; this is justified because for strong shear the poloidal field, giving rise to a toroidal mean current, is weak.

It is well known that the $\alpha$ effect is an extremely noisy quantity - especially in the case of large magnetic Reynolds numbers (Cattaneo \& Hughes 1996). The strong fluctuations are also clear from Fig. 6, where we plot $\alpha(t)$ both for open and closed boundaries. Note that $\alpha / u_{\text {rms }}$ fluctuates in time in the range \pm 0.2 about a much smaller average value of, e.g., -0.03 in the top panel. 

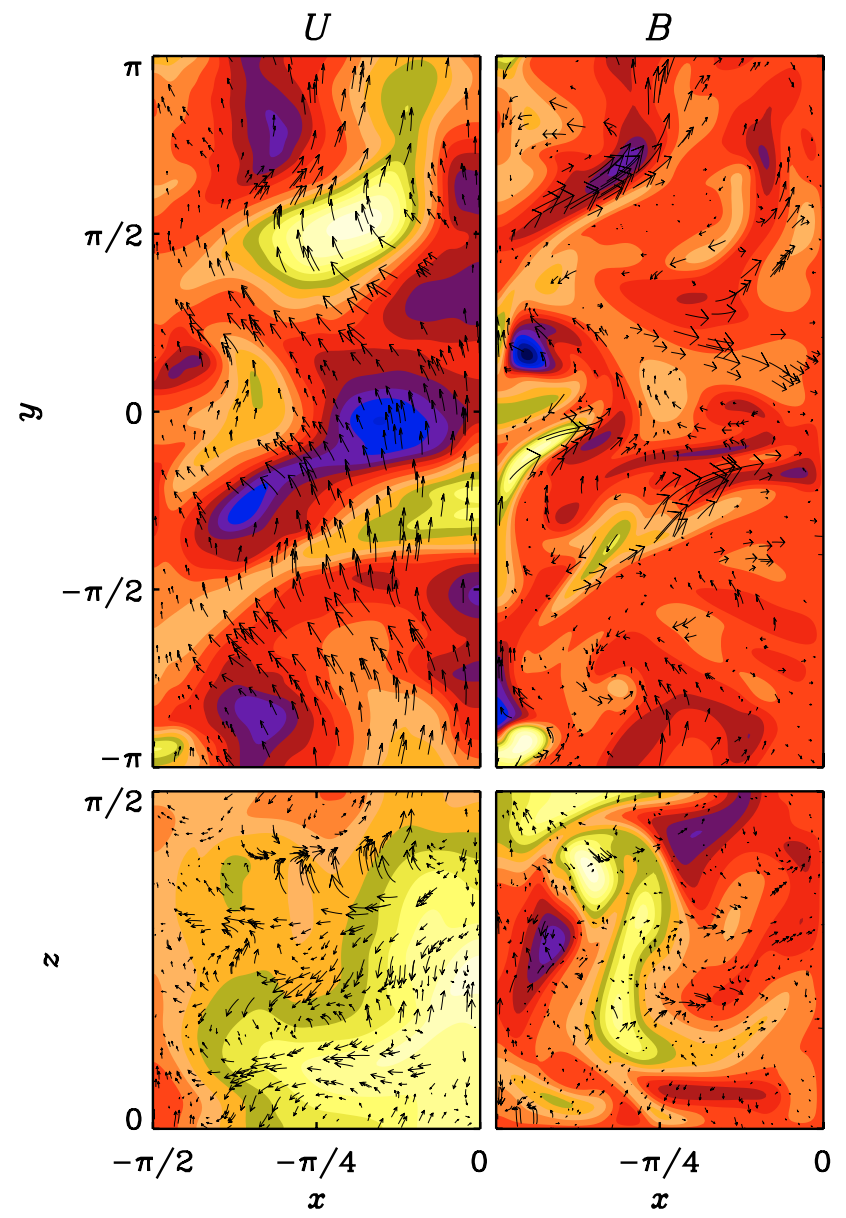

Fig. 5. Vectors of $\boldsymbol{U}$ and $\boldsymbol{B}$ in an $x y$ plane through $z=\pi / 4$, superimposed on a grey/color representation of their normal components, for a run with $R_{\mathrm{m}}=14$, open boundaries, shear, and negative helicity. Note that the velocity field is dominated by the toroidal shear flow.

As expected, $\alpha$ is negative when the helicity of the forcing is positive, and $\alpha$ changes sign when the helicity of the forcing changes sign. For $R_{\mathrm{m}} \gtrsim 14$ the magnitudes of $\alpha$ begin to be different in the two cases: $|\alpha|$ is larger when the helicity of the forcing is negative. In the sun, this corresponds to the sign of helicity in the northern hemisphere in the upper parts of the convection zone. This is here the relevant case, because the differential rotation pattern of our model also corresponds to the northern hemisphere.

There is a striking difference between the cases with open and closed boundaries which becomes particularly clear when comparing the averaged values of $\alpha$ for different magnetic Reynolds numbers; see Fig. 7. With closed boundaries $\alpha$ tends to zero like $R_{\mathrm{m}}^{-1}$, while with open boundaries $\alpha$ shows no such immediate decline; only for larger values of $R_{\mathrm{m}}$ there is possibly an asymptotic $\alpha \propto R_{\mathrm{m}}^{-1}$ dependence. There is also a clear difference between the cases with and without shear. In the absence of shear (dotted line in Fig. 7) $\alpha$ declines with increasing $R_{\mathrm{m}}$, even though for small values of $R_{\mathrm{m}}$ it is larger than with shear. This suggests that the presence of shear combined with open boundaries might be a crucial prerequisite of dynamos that saturate on a dynamical time scale.
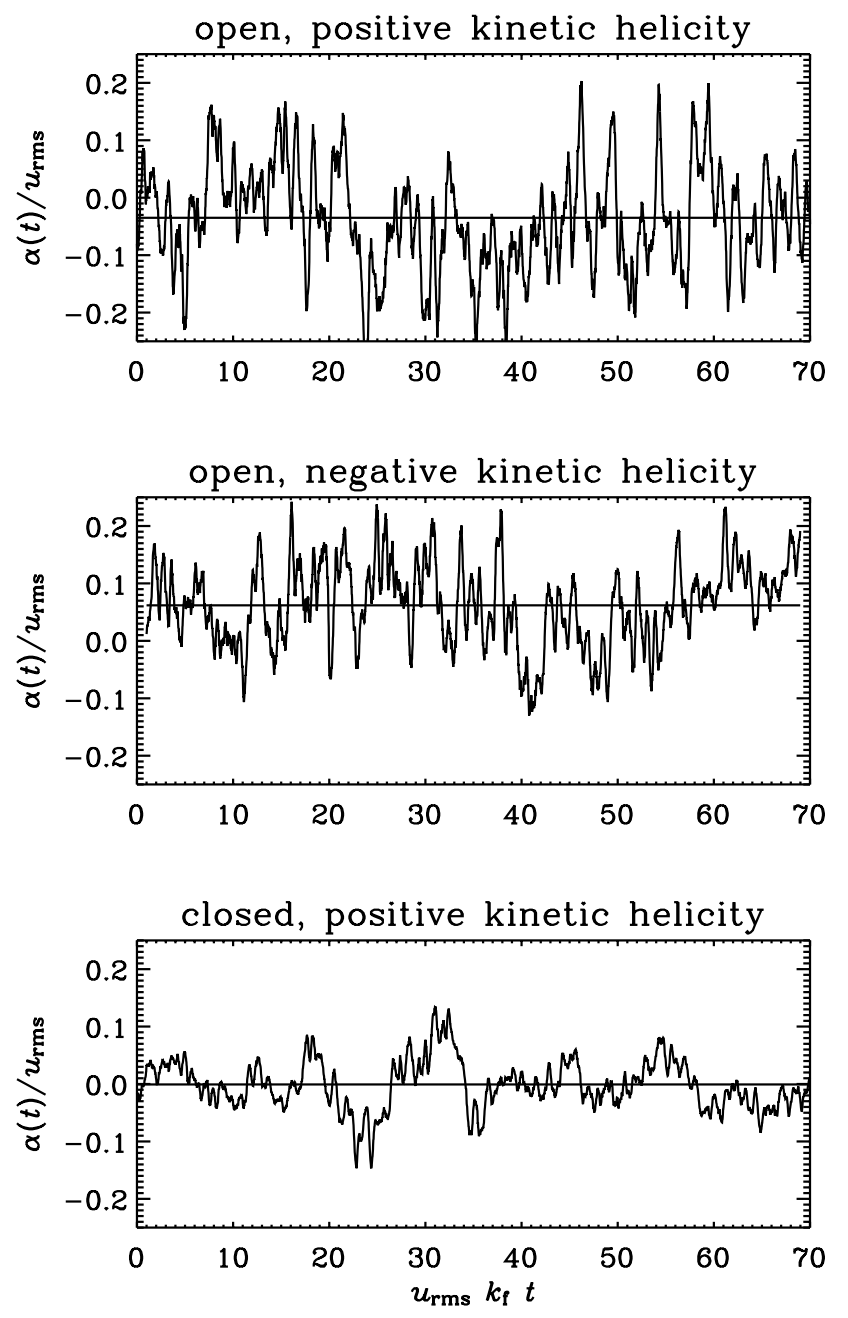

Fig. 6. Time traces of $\alpha(t)$ for runs with open boundaries (for both positive and negative kinetic helicity) and closed boundaries (for positive kinetic helicity). $R_{\mathrm{m}}=30$ in all cases.

The difference between open and closed boundaries will now be discussed in terms of a current helicity flux through the two open open boundaries of the domain.

\subsection{Current helicity flux}

It is suggestive to interpret the above results in terms of the dynamical $\alpha$ quenching model, where $\alpha$ is proportional to the difference between kinetic and current helicities (Pouquet et al. 1976), i.e.

$\alpha=-\frac{1}{3} \tau\left(\overline{\boldsymbol{\omega} \cdot \boldsymbol{u}}-\rho_{0}^{-1} \overline{\boldsymbol{j} \cdot \boldsymbol{b}}\right) \equiv \alpha_{\mathrm{K}}+\alpha_{\mathrm{M}}$.

Here, $\boldsymbol{\omega}=\boldsymbol{\nabla} \times \boldsymbol{u}$ is the small scale vorticity, $\boldsymbol{j}=\boldsymbol{\nabla} \times \boldsymbol{b} / \mu_{0}$ is the small scale current density, $\alpha_{\mathrm{K}}$ is the kinematic contribution to the $\alpha$ effect, and $\alpha_{\mathrm{M}}$ the magnetic contribution primarily responsible for the quenching of the $\alpha$ effect.

In order to obtain an expression for $\alpha_{\mathrm{M}}$ we begin with the evolution equation for $\overline{\boldsymbol{j} \cdot \boldsymbol{b}}$. In the absence of boundary conditions it has been advantageous to start with the evolution equation for magnetic helicity (because it is conserved) instead of current helicity (which is not conserved); see 


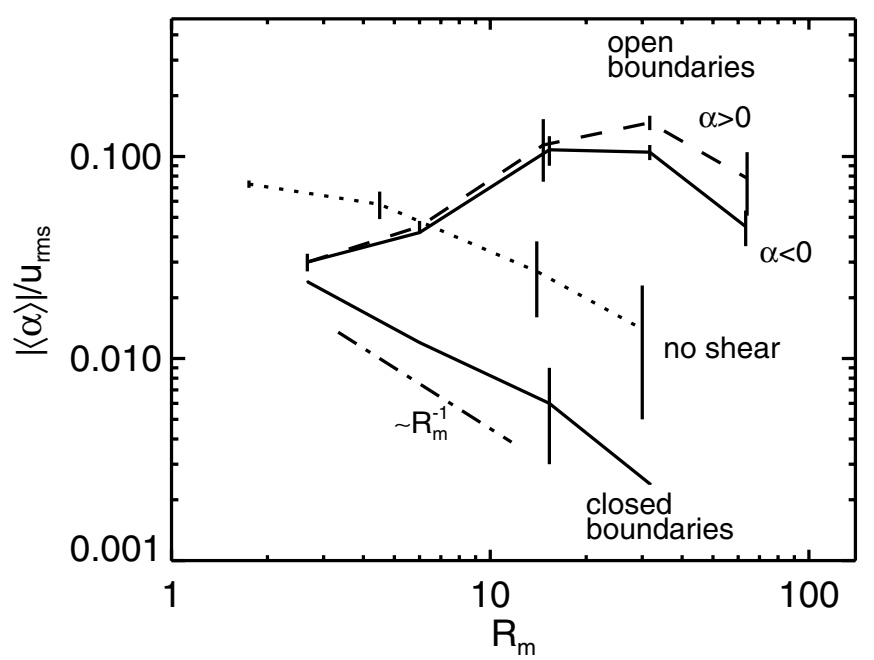

Fig. 7. Dependence of $|\langle\alpha\rangle| / u_{\mathrm{rms}}$ on $R_{\mathrm{m}}$ for open and closed boundaries. The case with open boundaries and negative helicity is shown as a dashed line. Note that for $R_{\mathrm{m}} \approx 30$ the $\alpha$ effect is about 30 times smaller when the boundaries are closed. The dotted line gives the result with open boundaries but no shear. The vertical lines indicate the range obtained by calculating $\alpha$ using only the first and second half of the time interval.

Kleeorin \& Ruzmaikin (1982), Kleeorin et al. (1995), Blackman \& Brandenburg (2002). In the case of open boundary conditions, this approach becomes cumbersome, because one has to consider the gauge-invariant relative magnetic helicity (Berger \& Field 1984). Furthermore, the concept of a density of magnetic helicity is not meaningful, because it would depend on the gauge. In order to avoid these problems it is advantageous consider the current helicity equation (Subramanian \& Brandenburg 2004, Brandenburg \& Subramanian 2004). Apart from this technicality, the following derivation is similar to that of Blackman \& Brandenburg (2002) for the case without current helicity flux.

Using the evolution equation, $\partial \boldsymbol{b} / \partial t=-\boldsymbol{\nabla} \times \boldsymbol{e}$, for the fluctuating magnetic field, where $\boldsymbol{e}=\boldsymbol{E}-\overline{\boldsymbol{E}}$ is the small scale electric field and $\overline{\boldsymbol{E}}=\eta \overline{\boldsymbol{J}}-\overline{\boldsymbol{E}}$ the mean electric field, we can derive the equation

$\frac{\partial}{\partial t} \overline{\boldsymbol{j} \cdot \boldsymbol{b}}=-2 \overline{\boldsymbol{e} \cdot \boldsymbol{c}}-\boldsymbol{\nabla} \cdot \overline{\mathcal{F}}_{C}^{\mathrm{SS}}$

where

$\overline{\mathcal{F}}_{C}^{S S}=\overline{2 e \times j}+\overline{(\nabla \times e) \times b}$

is the current helicity flux from the small scale field, and $c=$ $\boldsymbol{\nabla} \times \boldsymbol{j}$ the curl of the small scale current density, $\boldsymbol{j}=\boldsymbol{J}-\overline{\boldsymbol{J}}$. In the isotropic case, $\overline{\boldsymbol{e} \cdot \boldsymbol{c}} \approx k_{\mathrm{f}}^{2} \overline{\boldsymbol{e} \cdot \boldsymbol{b}}$, where $k_{\mathrm{f}}$ is the typical wavenumber of the fluctuations, here assumed to be the forcing wavenumber. Ignoring the effect of the mean flow on $\overline{\mathcal{E}}$ (as is usually done; but see Krause \& Rädler 1980), we obtain

$\overline{\boldsymbol{e} \cdot \boldsymbol{b}} \approx-\overline{\left(\boldsymbol{u} \times \boldsymbol{B}_{0}\right) \cdot \boldsymbol{b}}+\eta \overline{\boldsymbol{j} \cdot \boldsymbol{b}}=\overline{\mathcal{E}} \cdot \overline{\boldsymbol{B}}+\eta \overline{\boldsymbol{j} \cdot \boldsymbol{b}}$,

where we have used $\overline{\boldsymbol{u} \times \boldsymbol{b}}=\overline{\boldsymbol{\varepsilon}}$ and $\boldsymbol{B}_{0}=\overline{\boldsymbol{B}}$. Using standard expressions for the turbulent magnetic diffusivity, $\eta_{\mathrm{t}}=\frac{1}{3} \tau u_{\mathrm{rms}}^{2}$, and the equipartition field strength, $B_{\text {eq }}=\sqrt{\mu_{0} \rho} u_{\mathrm{rms}}$, we eliminate $\tau$ via

$\frac{1}{3} \tau \rho_{0}^{-1}=\eta_{\mathrm{t}} / B_{\text {eq }}^{2}$.

This leads to an explicitly time dependent formula for $\alpha$,

$\frac{\partial \alpha}{\partial t}=-2 \eta_{\mathrm{t}} k_{\mathrm{f}}^{2}\left(\frac{\overline{\mathcal{E}} \cdot \overline{\boldsymbol{B}}+\frac{1}{2} k_{\mathrm{f}}^{-2} \boldsymbol{\nabla} \cdot \overline{\mathcal{F}}_{C}^{\mathrm{SS}}}{B_{\mathrm{eq}}^{2}}+\frac{\alpha-\alpha_{\mathrm{K}}}{R_{\mathrm{m}}}\right)$.

This equation is similar to that of Kleeorin et al. (2000-2003) who considered the flux of magnetic helicity instead of current helicity.

Making the adiabatic approximation, i.e. putting the rhs of Eq. (20) to zero, one arrives at the algebraic steady state quenching formula $(\partial \alpha / \partial t=0)$

$\alpha=\frac{\alpha_{\mathrm{K}}+R_{\mathrm{m}}\left(\eta_{\mathrm{t}} \mu_{0} \overline{\boldsymbol{J}} \cdot \overline{\boldsymbol{B}}-\frac{1}{2} k_{\mathrm{f}}^{-2} \boldsymbol{\nabla} \cdot \overline{\mathcal{F}}_{\mathrm{C}}^{\mathrm{SS}}\right) / B_{\mathrm{eq}}^{2}}{1+R_{\mathrm{m}} \overline{\boldsymbol{B}}^{2} / B_{\mathrm{eq}}^{2}}$.

Furthermore, if the mean field is defined as an average over the whole box, then $\overline{\boldsymbol{B}} \equiv \boldsymbol{B}_{0}=$ const, so $\overline{\boldsymbol{J}}=0$ and Eq. (21) reduces to

$\alpha=\frac{\alpha_{\mathrm{K}}-\frac{1}{2} k_{\mathrm{f}}^{-2} R_{\mathrm{m}} \boldsymbol{\nabla} \cdot \overline{\mathcal{F}}_{C}^{\mathrm{SS}} / B_{\mathrm{eq}}^{2}}{1+R_{\mathrm{m}} \boldsymbol{B}_{0}^{2} / B_{\mathrm{eq}}^{2}}$.

This expression applies to the present case, because we consider only the statistically steady state and we also define the mean field as a volume average.

For closed boundaries, $\boldsymbol{\nabla} \cdot \overline{\mathcal{F}}_{C}^{\text {SS }}=0$, and so Eq. (22) clearly reduces to a catastrophic quenching formula, i.e. $\alpha$ vanishes in the limit of large magnetic Reynolds numbers as

$\alpha^{\text {(closed) }}=\frac{\alpha_{\mathrm{K}}}{1+R_{\mathrm{m}} \boldsymbol{B}_{0}^{2} / B_{\mathrm{eq}}^{2}} \rightarrow R_{\mathrm{m}}^{-1} \quad\left(\right.$ for $\left.R_{\mathrm{m}} \rightarrow \infty\right)$.

The $R_{\mathrm{m}}^{-1}$ dependence suggested by Eq. (23) is confirmed by the simulations (compare with the dash-dotted line in Fig. 7). On the other hand, for open boundaries the limit $R_{\mathrm{m}} \rightarrow \infty$ gives

$\alpha^{(\text {open })} \rightarrow-\left(\boldsymbol{\nabla} \cdot \overline{\mathcal{F}}_{C}^{\mathrm{SS}}\right) /\left(2 k_{\mathrm{f}}^{2} \boldsymbol{B}_{0}^{2}\right) \quad\left(\right.$ for $\left.R_{\mathrm{m}} \rightarrow \infty\right)$,

which shows that losses of negative helicity, as observed in the northern hemisphere of the sun, would enhance a positive $\alpha$ effect (Kleeorin et al. 2000). In the simulations, the current helicity flux is found to be independent of the magnetic Reynolds number. This explains why the $\alpha$ effect no longer shows the catastrophic $R_{\mathrm{m}}^{-1}$ dependence (see Fig. 7).

\subsection{Estimates for the Vishniac-Cho flux}

Theoretical estimates for magnetic helicity fluxes have been proposed by Kleeorin et al. (2000) and Vishniac \& Cho (2001). The two fluxes are rather different. The expression of Vishniac \& Cho (2001) has been confirmed independently and can be written in the form (Subramanian \& Brandenburg 2004; see also the review by Brandenburg \& Subramanian 2004)

$\overline{\mathcal{F}}_{k}^{\mathrm{VC}}=-4 \tau \overline{\omega_{i} \nabla_{j} u_{k}} \bar{B}_{i} \bar{B}_{j}$. 

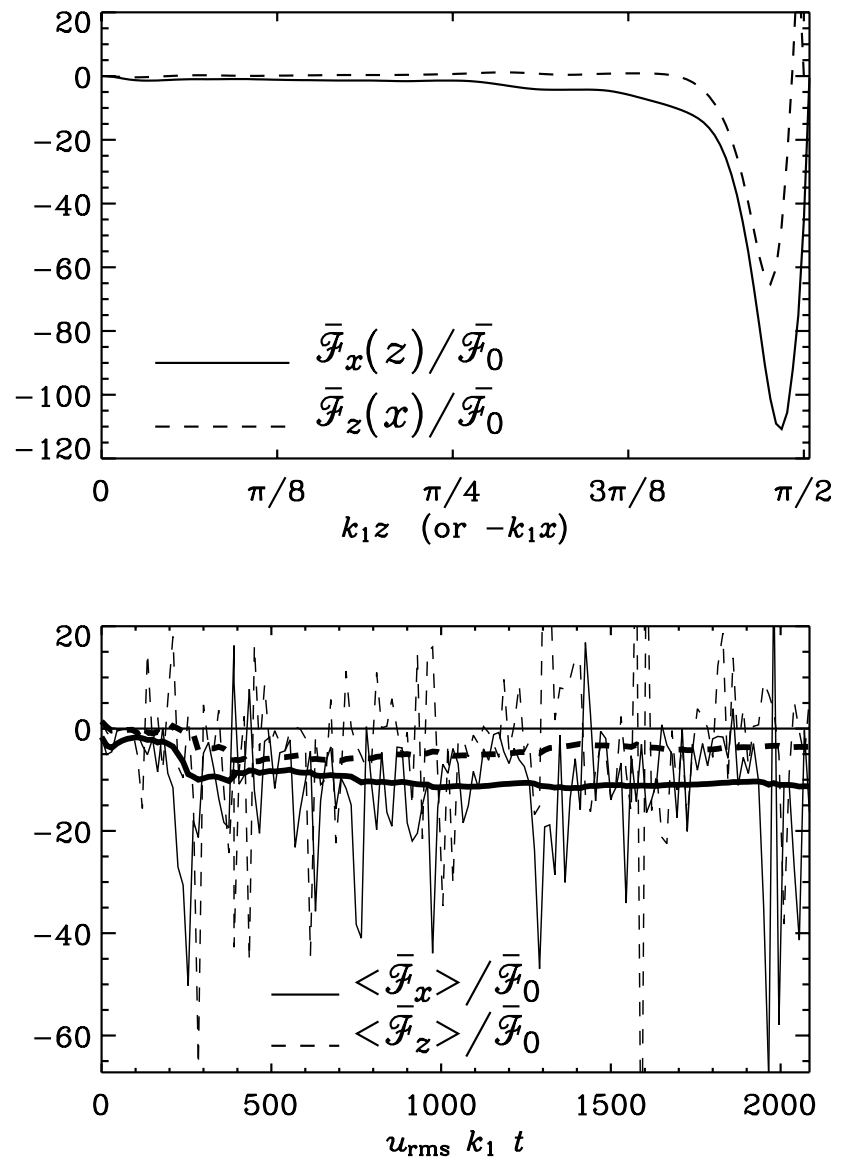

Fig. 8. The Vishniac-Cho flux on the outer surface $\left(\overline{\mathcal{F}}_{x}^{\mathrm{VC}}\right)$ and at the equator $\left(\overline{\mathcal{F}}_{z}^{\mathrm{VC}}\right)$, averaged over the $y$ direction, for the run with $\alpha>$ 0 and $R_{\mathrm{m}}=14$. In the upper panel the flux is also averaged over $t$ and shown as a function of $z$ and $x$, respectively. In the lower panel, the $x$ and $z$ components of the fluxes are averaged over the $z$ and $x$ directions, respectively, and shown as functions of $t$. The fat solid and dashed lines denote the running means for the two functions.

In Fig. 8 we plot the profiles of this flux, averaged in the $y$ direction, on the two open boundaries. We also show the time evolution of the averaged fluxes on the two open boundaries. It turns out that the magnitude of the two fluxes is large compared with $\overline{\mathcal{F}}_{0} \equiv u_{\mathrm{rms}} k_{\mathrm{f}} B_{0}^{2}$, but the fluxes also fluctuate strongly in time, so it is important to average over long times. Furthermore, there is a clear tendency for the difference between incoming flux at the equator $\left(\overline{\mathcal{F}}_{z}^{\mathrm{VC}}\right.$, dashed line) and outgoing fluxes at outer surface $\left(\overline{\mathcal{F}}_{x}^{\mathrm{VC}}\right.$, solid line) to cancel partially, giving a smaller net flux. Nevertheless, since $\left|\overline{\mathcal{F}}_{x}^{\mathrm{VC}}\right|>\left|\overline{\mathcal{F}}_{z}^{\mathrm{VC}}\right|$, the net outgoing flux is negative, as expected for the northern hemisphere.

\subsection{Large scale current helicity flux}

In earlier work (Brandenburg \& Dobler 2001) it was reported that the contribution to the magnetic helicity flux was outweighed by a much larger flux from the large scale field. In

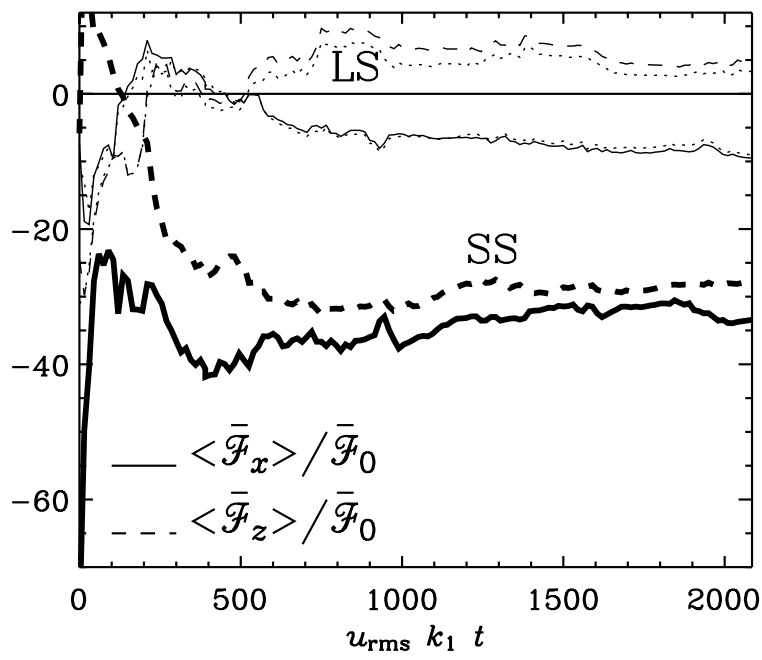

Fig. 9. Normal components of the current helicity flux on the outer surface $\left(\overline{\mathcal{F}}_{x}\right)$ and at the equator $\left(\overline{\mathcal{F}}_{z}\right)$, averaged over the corresponding surfaces, for the same run as in Fig. 8. The fat lines denote the fluxes from the small scale field, $\overline{\mathcal{F}}_{C}^{\mathrm{SS}}$, while the thin lines denote the fluxes form the large scale field, $\overline{\mathcal{F}}_{C}^{\text {LS }}$. The dotted lines near the two $\overline{\mathcal{F}}_{C}^{\text {LS }}$ curves show the result of the approximation (27).

the present paper we work instead with the current helicity, and the current helicity flux from the large scale field is

$\overline{\mathcal{F}}_{C}^{\mathrm{LS}}=2 \overline{\boldsymbol{E}} \times \overline{\boldsymbol{J}}+(\boldsymbol{\nabla} \times \overline{\boldsymbol{E}}) \times \overline{\boldsymbol{B}}$.

For the vertical field condition, see Eqs. (6) and (7), the second term in Eq. (26) vanishes. Assuming isotropy, the contribution from the first term involves $\overline{\mathcal{E}}=\alpha \overline{\boldsymbol{B}}-\eta_{\mathrm{t}} \overline{\boldsymbol{J}}$, but this does not contribute either, because $\alpha \overline{\boldsymbol{B}} \times \overline{\boldsymbol{J}}$ does not have a normal component on the boundaries, and $\eta_{\mathrm{t}} \overline{\boldsymbol{J}} \times \overline{\boldsymbol{J}}=0$. In the first term only the mean flow term contributes, so we get

$\overline{\mathcal{F}}_{C}^{\mathrm{LS}} \approx-2(\overline{\boldsymbol{U}} \times \overline{\boldsymbol{B}}) \times \overline{\boldsymbol{J}}=-2\left(\bar{J}_{y} \bar{U}_{y}\right) \overline{\boldsymbol{B}}$.

Inspection of the data suggests that this is indeed a good approximation and that therefore even in the simulation the normal component of $\overline{\mathcal{E}} \times \overline{\boldsymbol{J}}$ is nearly vanishing on the boundary; see Fig. 9. Both on the outer surface and on the equator $\overline{\mathcal{F}}_{C}^{\text {LS }}$ is such that it corresponds to a loss of negative current helicity.

In Fig. 9 we also show the small scale current helicity fluxes on the two boundaries (fat lines). There is a tendency for the difference between incoming flux at the equator (dotted line) and outgoing fluxes at outer surface (solid line) to cancel, but the net outgoing flux is again negative. The flux for the total field is approximately four times larger than what is accounted for by the Vishniac-Cho flux. This might indicate that there is either another contribution to the current helicity flux, or that the $\tau$ in the Vishniac-Cho flux is underestimated.

\subsection{Application to the sun}

The purpose of this section is to put some real numbers into the expression for the current helicity flux. Our simulations have 




Fig. 10. Sketch illustrating the directions of large scale (LS) and small scale (SS) negative current helicity fluxes and their approximate magnitudes (in units of $\overline{\mathcal{F}}_{0}$ ). Note that at the outer surface negative current helicity is ejected both via small and large scale fields, while at the equator the contributions from small and large scale fields have opposite sign. The small scale losses at surface and equator partially cancel, giving a net loss of negative current helicity of only about $4 \overline{\mathcal{F}}_{0}$.

shown that a reasonable estimate for the current helicity flux at the outer surface is

$\overline{\mathcal{F}}_{C}^{\mathrm{SS}} \approx 30 u_{\mathrm{rms}} k_{\mathrm{f}} B_{0}^{2}$.

Applying this to the sun using $u_{\mathrm{rms}} \approx 50 \mathrm{~m} / \mathrm{s}$ for the rms velocity in the deeper parts of the convection zone, $k_{\mathrm{f}} \approx 10^{-9} \mathrm{~cm}^{-1}$ based on the inverse mixing length, and $B_{0} \approx 3 \mathrm{G}$ for the mean field at the solar surface, we have $\overline{\mathcal{F}}_{C}^{S S} \approx 10^{-3} \mathrm{G}^{2} / \mathrm{s}$. The current helicity flux integrated over the northern hemisphere of the sun is then $4 \times 10^{19} \mathrm{G}^{2} \mathrm{~cm}^{2} \mathrm{~s}^{-1}$. Integrated over the $11 \mathrm{yr}$ solar cycle we have $10^{28} \mathrm{G}^{2} \mathrm{~cm}^{2}$.

For the sun only magnetic helicity fluxes have been determined. As a rough estimate we may use $\overline{\mathcal{F}}_{H}^{\text {SS }} \approx k_{\mathrm{f}}^{-2} \overline{\mathcal{F}}_{C}^{\text {SS }}$ for the magnetic helicity flux. Using the same estimate for $k_{\mathrm{f}}$ as above we obtain about $10^{46} \mathrm{Mx}^{2}$ over the $11 \mathrm{yr}$ solar cycle. This is indeed comparable to the magnetic helicity fluxes estimated by Berger \& Ruzmaikin (2000) and DeVore (2000).

We emphasize that, in the present context, "small scale" means $2 \pi / k_{\mathrm{f}} \approx 60 \mathrm{Mm}$, i.e. about one pressure scale height at the bottom of the convection zone. By contrast, "large scale" refers to length scales of the order of several hundred $\mathrm{Mm}$, which is typically beyond the scale captured in the usual vector magnetograms.

\section{Conclusions}

The present simulations give a clear indication that the proposed set-up with open boundaries and shear can alleviate the catastrophic quenching problem in mean field dynamo theory. Already in the absence of shear the resulting $\alpha$ effect can be larger when open boundary conditions are used, but there $\alpha$ still decreases with increasing magnetic Reynolds number. In the presence of shear, alleviated catastrophic quenching is associated with a net loss of small scale current helicity. At the equator, negative small scale current helicity flows into the northern hemisphere, but there is an even larger negative small scale current helicity flows ejected at the outer surface. This results in a net loss of negative current helicity from the northern hemisphere, and corresponds to an integrated magnetic helicity flux of about $10^{46} \mathrm{Mx}^{2}$ over the $11 \mathrm{yr}$ solar cycle.

Although our results are certainly encouraging, they must still be considered preliminary. First of all, we have still only considered a relatively limited range of magnetic Reynolds numbers; larger values are necessary before one can tell whether or not an asymptotic $R_{\mathrm{m}}$ dependence still develops for larger values of $R_{\mathrm{m}}$. Secondly, turbulence simulations of a dynamo are necessary to show that the electromotive force that here is interpreted in terms of an $\alpha$ effect is indeed capable of generating large scale magnetic field of the type shown in Sect. 3. Of particular importance is the question whether the dynamo is oscillatory (as expected from our mean field calculations) and whether the cycle frequency is independent of $R_{\mathrm{m}}$. Especially at large values of $R_{\mathrm{m}}$ the direct approach tends to be advantageous compared to calculating $\alpha$ in the presence of an imposed field, because the resulting $\alpha$ is always much more noisy than the actual mean field obtained in a simulation (B01).

Another aspect to keep in mind is the fact that the correct boundary conditions for the solar dynamo are certainly more complicated than the vertical field condition adopted here. There are good reasons to believe that the sun loses significant amounts of magnetic and current helicity via coronal mass ejections (DeVore 2000; Démoulin et al. 2002a,b; Gibson et al. 2002; see also Blackman \& Brandenburg 2003). The losses via coronal mass ejections are not easy to model within the present approach. An obvious possibility is to include an outer layer that resembles some important aspects of the solar corona (low density and hence low plasma beta). It is as yet unclear to which extent a spherical geometry is important. The solar wind, for example, cannot be modeled in Cartesian geometry, and in some sense coronal mass ejections are just a particularly bursty and localized manifestation of the solar wind. It may therefore be worthwhile to consider the effects of boundaries in global simulations.

Acknowledgements. We thank Eric Blackman, Detlef Elstner, and Kandu Subramanian for extended discussions and detailed suggestions. C. S. thanks NORDITA for hospitality. The Danish Center for Scientific Computing is acknowledged for granting time on the Linux cluster in Odense (Horseshoe).

\section{References}

Arlt, R., \& Brandenburg, A. 2001, A\&A, 380, 359

Berger, M. 1984, Geophys. Astrophys. Fluid Dyn., 30, 79

Berger, M., \& Field, G. B. 1984, JFM, 147, 133

Berger, M., \& Ruzmaikin, A. 2000, JGR, 105, 10481

Blackman, E. G., \& Brandenburg, A. 2002, ApJ, 579, 359

Blackman, E. G., \& Brandenburg, A. 2003, ApJ, 584, L99

Blackman, E. G., \& Field, G. F. 2000a, ApJ, 534, 984

Blackman, E. G., \& Field, G. F. 2000b, MNRAS, 318, 724

Brandenburg, A. 2001, ApJ, 550, 824 (B01) 
Brandenburg, A., \& Dobler, W. 2001, A\&A, 369, 329

Brandenburg, A., \& Subramanian, K. 2004, Phys. Rep., submitted [arXiv: astro-ph/0405052]

Brandenburg, A., Nordlund, Å., Pulkkinen, P., Stein, R. F., \& Tuominen, I. 1990, A\&A, 232, 277

Brandenburg, A., Bigazzi, A., \& Subramanian, K. 2001, MNRAS, 325,685

Brandenburg, A., Dobler, W., \& Subramanian, K. 2002, AN, 323, 99

Cattaneo F., \& Hughes D. W. 1996, PRE, 54, R4532

Choudhuri, A. R., Schüssler, M., \& Dikpati, M. 1995, A\&A, 303, L29

DeVore, C. R. 2000, ApJ, 539, 944

Démoulin, P., Mandrini, C. H., van Driel-Gesztelyi, L., Lopez Fuentes, M. C., \& Aulanier, G. 2002, Sol. Phys., 207, 87

Démoulin, P., Mandrini, C. H., van Driel-Gesztelyi, L., et al. 2002, ApJ, 382, 650

Dikpati, M., \& Gilman, P. A. 2001, ApJ, 559, 428

Durney, B. R. 1995, Sol. Phys., 166, 231

Field, G. B., \& Blackman, E. G. 2002, ApJ, 572, 685

Gibson, S. E., Fletcher, L., Del Zanna, G., et al. 2002, ApJ, 574, 1021 Ji, H. 1999, PRL, 83, 3198

Kleeorin, N. I., \& Ruzmaikin, A. A. 1982, Magnetohydrodynamics, 18,116

Kleeorin, N. I., Rogachevskii, I., \& Ruzmaikin, A. 1995, A\&A, 297, 159
Kleeorin, N. I., Moss, D., Rogachevskii, I., \& Sokoloff, D. 2000, A\&A, 361, L5

Kleeorin, N. I., Moss, D., Rogachevskii, I., \& Sokoloff, D. 2002, A\&A, 387, 453

Kleeorin, N. I., Moss, D., Rogachevskii, I., \& Sokoloff, D. 2003, A\&A, 400, 9

Krause, F., \& Rädler, K.-H. 1980, Mean-Field Magnetohydrodynamics and Dynamo Theory (Berlin: Akademie-Verlag; also Oxford: Pergamon Press)

Moffatt, H. K. 1978, Magnetic Field Generation in Electrically Conducting Fluids (Cambridge: Cambridge University Press)

Ossendrijver, M., Stix, M., \& Brandenburg, A. 2001, A\&A, 376, 713

Parker, E. N. 1979, Cosmical Magnetic Fields (Oxford: Clarendon Press)

Pouquet, A., Frisch, U., \& Léorat, J. 1976, JFM, 77, 321

Roberts, P. H. 1972, Phil. Trans. Roy. Soc., A272, 663

Roberts, P. H., \& Stix, M. 1972, A\&A, 18, 453

Seehafer, N. 1996, PRE, 53, 1283

Subramanian, K. 2002, Bull. Astr. Soc. India, 30, 715

Subramanian, K., \& Brandenburg, A. 2004, [arXiv: astro-ph/0408020]

Vishniac, E. T., \& Cho, J. 2001, ApJ, 550, 752

Zeldovich, Ya. B., Ruzmaikin, A. A., Sokoloff, D. D. 1983, Magnetic fields in astrophysics (New York: Gordon \& Breach) 